\title{
Tumor-Treating Fields at EMBC 2019: A Roadmap to Developing a Framework for TTFields Dosimetry and Treatment Planning
}

\author{
Ze'ev Bomzon, Cornelia Wenger, Martin Proescholdt, and Suyash Mohan
}

\section{Introduction}

Tumor-treating fields (TTFields) are electric fields with intensities of $1-5 \mathrm{~V} / \mathrm{cm}$ in the frequency range of $100-500 \mathrm{kHz}$ known to inhibit the growth of cancerous tumors. TTFields have been approved for the treatment of glioblastoma multiforme (GBM) since 2011 [19-21]. Recently, the therapy was FDA-approved for the treatment of malignant pleural mesothelioma (MPM) [4]. TTFields are delivered noninvasively through two pairs of transducer arrays that are placed on the patient's skin in close proximity to the tumor (see Fig. 1). At any instance, only one pair of arrays is used to create the field, while the second pair is switched off. The pairs of arrays are placed such that the fields created are roughly orthogonal, and switching of the active arrays occurs about once per second. This results in the creation of an alternating electric field, which switches direction periodically. The field is generated by a portable field generator. Treatment is continuous as analysis of clinical data has shown a positive connection between device usage (fraction of time patient is on therapy) and patient outcomes [2].

Preclinical studies have shown that the antimitotic effect of TTFields is frequency- and intensity-dependent. The inhibitory effect on different cell types is observed at cell-specific frequencies $[6,9,10]$, and the higher the intensity of the

\footnotetext{
Z. Bomzon $(\bowtie) \cdot$ C. Wenger

Novocure Ltd., Haifa, Israel

e-mail: zbomzon@novocure.com

M. Proescholdt

Department of Neurosurgery, University Regensburg Hospital Medical Center, Regensburg, Germany

S. Mohan

Department of Radiology, Division of Neuroradiology, Perelman School of Medicine

University of Pennsylvania, Philadelphia, PA, USA
} 

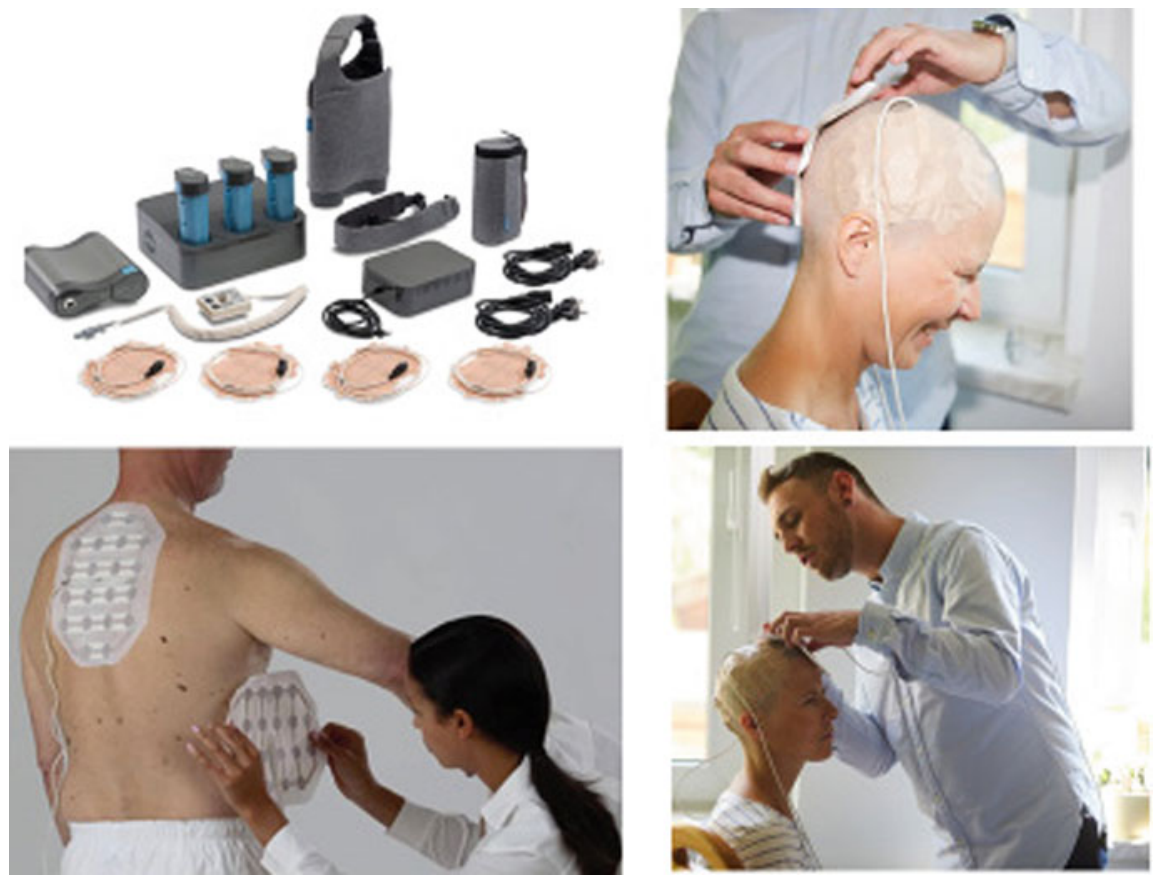

Fig. 1 Top left image shows Optune ${ }^{\mathrm{TM}}$ device used to deliver TTFields to the brain. The device comprises a portable battery-powered field generator, connected to four transducer arrays which are placed on the scalp as shown in top and bottom-right images. The image in the bottom-left corner shows placement of transducer arrays for treatment of thoracic tumors with TTFields

field, the stronger the inhibitory effect. As mentioned above, the effect of TTFields is also time-dependent, with higher usage associated with improved patient survival [18]. Posthoc analysis of the EF-14 trials showed overall survival for patients treated with TTFields+temozolomide (TMZ) with usage of $90 \%$ or more was 24 months compared to 16.03 months in patients treated with TMZ alone [18]. More recently, a study by Ballo et al. [2] showed that in newly diagnosed GBM patients, survival correlated with TTFields dose delivered to the tumor bed. Dose was defined as power loss density multiplied by usage. These findings suggest that patient outcome could be significantly improved with rigorous treatment planning, in which numerical simulations are used to identify array layouts that optimize delivery of TTFields to the tumor bed. The plan could be adapted periodically as the tumor evolves to maximize the effect of treatment in regions where tumor progression occurs.

Performing such adaptive planning in a practical and meaningful manner requires a rigorous and scientifically proven framework defining TTFields dose and showing how dose distribution influences disease progression in different malignancies (TTFields dosimetry). The adaptive planning also requires a set of principles on how best to perform treatment planning, along with numerical methods and algorithms devised to optimize therapy based on the principles mentioned above. The 
principles should be derived from our understanding of TTFields dosimetry and how dose distributions influence disease. An effective treatment planning strategy also requires quality assurance and uncertainty analysis to understand how uncertainties in the model, numerical solver, and positions of the array influence the field distribution to create a quality assurance system to ensure that the plan is adhered to within the allowed uncertainties.

At EMBC 2019, several talks discussing key components related to TTFields dosimetry and treatment planning were presented. The purpose of this manuscript is to provide a short overview of this work and discuss how it sets the foundations for the emerging field of TTFields dosimetry and treatment planning.

\section{An Outline for TTFields Dosimetry and Treatment Planning}

Figure 2 is a flow chart describing the steps required in order to realize an effective scheme for TTFields treatment planning.

The first step in the process is clinical evaluation and contouring. In this step, the planning physician examines imaging data of the patient, identifies regions of active

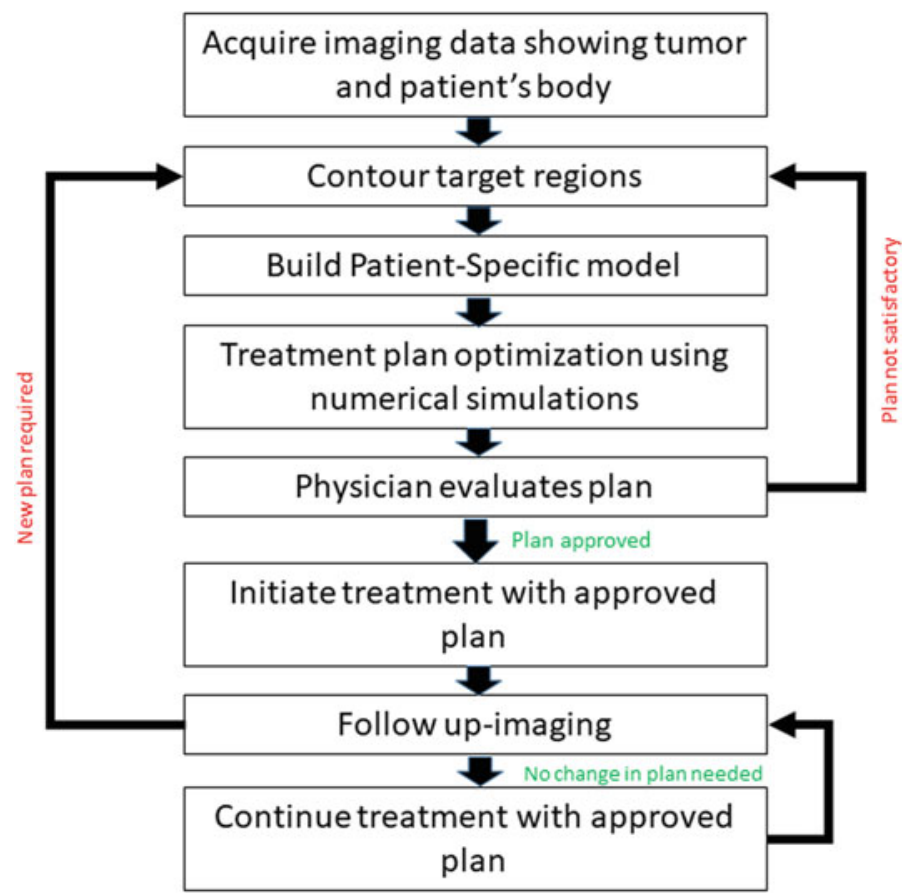

Fig. 2 Flowchart describing the steps involved in treatment planning and how these could be integrated into an effective workflow in the clinic 
tumor, and selects the target regions in which TTFields dose should be optimized. The physician may also identify areas to avoid, like areas on the skin over which transducer arrays should not be placed. Next, the imaging data are used to create a patient-specific computational model, which can be used to simulate delivery of TTFields to the patient. In the context of TTFields treatment planning, the model involves the creation of a three-dimensional representation of the patient, in which electric conductivity is assigned to each point. The model, target regions, and avoidance areas are inputs for an optimization algorithm that seeks to find a transducer array layout that optimizes the dose in the target regions while avoiding placement of transducer arrays over the avoidance structures. The optimization algorithm will typically entail iterative use of a numerical solver that simulates delivery of TTFields to the patient for different array layouts. The output from this process will be an optimal array layout for treating the patient, as well as quantitative and visual aids that enable the physician to evaluate the quality of the plan. These aids could include color maps describing the field distribution within the patient's body and dose-volume histogram (DVH) describing the distribution of TTFields dose within the target regions and within other areas of interest. Once a plan that the physician deems satisfactory has been generated, the patient is instructed on how to place the transducer arrays on their body, and treatment commences. Patient followups occur periodically. During these follow-ups, additional imaging of the patient may be acquired as physician assesses for disease progression. The new imaging data may demonstrate regions in which the tumor has responded to therapy and/or regions in which tumor has progressed. Depending on patient's patterns of response and progression, the physician may decide to re-plan in order to enhance treatment to new target regions.

Three key components required to establish an effective framework for TTFields treatment planning are:

TTFields Dosimetry: An understanding of how to define TTFields dose accurately and an understanding on how TTFields dose distributions influence disease progression and patient outcomes.

Patient-Specific Model Creation: An ability to accurately calculate field distributions within patients in a quick and reliable manner is crucial for TTFields treatment planning. This in turn requires an ability to build 3D patient-specific models in which conductivity at each point within the model is well-defined, so that accurate dose distributions can be calculated.

Advanced Imaging for Monitoring Response to Therapy: Imaging technologies that enable accurate mapping of tumors and changes that occur within the tumor.

Below is a discussion of work presented at EMBC 2019, which touches on these three topics. 


\section{TTFields Dosimetry}

As mentioned above, preclinical research has shown that the effect of TTFields on cancer cells depends on the frequency of the field, its intensity, and the duration of exposure to the fields. The EF-14 trial compared patient outcome in patients treated with chemoradiation+TTFields with outcome in patients treated with chemoradiation alone. Post hoc analysis of this trial has shown that patient outcome positively correlates with device usage (\% of time on active treatment) [2].

More recently, Ballo et al. [2] published a study in which they defined TTFields dose as power loss density multiplied by usage and showed that within the EF-14 trial population, patients who received higher doses to the tumor bed exhibited overall improvement. Bomzon et al. presented a summary of this work at EMBC 2019 [3].

A total of $N=340$ patients who received TTFields as part of the EF-14 trial were included in the study. Realistic head models of the patients were derived from T1-contrast-enhanced images captured at baseline using a previously described method [24]. The transducer array layout on each patient was obtained from EF-14 records, and average usage and average electrical current delivered to the patient during the first 6 months of treatment were derived from log files of the TTFields devices used by patients. Finite element simulations of TTFields delivery to the patients were performed using Sim4Life (ZMT Zurich, Switzerland). The average field intensity, power loss density, and dose density within a tumor bed comprising the gross tumor volume and the 3-mm-wide peritumoral boundary zone were calculated. The values of average field intensity, power loss density, and dose density that divided the patients into two groups with the most statistically significant difference in OS were identified. ${ }^{1}$

Figure 3 shows Kaplan-Meier curves for overall survival (OS) when dividing the patients into two groups according to TTFields dose. The median OS (and PFS data not shown) was significantly longer when average TTFields dose in the tumor bed was $>0.77 \mathrm{~mW} / \mathrm{cm}^{3}$ : OS (25.2 vs 20.4 months, $\left.p=0.003, \mathrm{HR}=0.611\right)$ and PFS (8.5 vs 6.7 months, $p=0.02, \mathrm{HR}=0.699$ ). In similar analysis, dividing the patients according to TTFields intensity yielded that median OS and PFS were longer when average TTFields intensity at the tumor bed was $>1.06 \mathrm{~V} / \mathrm{cm}$ OS $(24.3 \mathrm{vs}$ 21.6 months, $p=0.03, \mathrm{HR}=0.705)$ and PFS (8.1 vs 7.9 months, $p=0.03$, $\mathrm{HR}=0.721)$.

This work sets a foundation for defining TTFields dose. It shows that TTFields dose can be defined in terms of power and usage and that delivery of higher doses of

\footnotetext{
${ }^{1}$ Defining dose for TTFields therapy is important to remember that TTFields are delivered by two sets of arrays, with the field direction switching direction every second. Thus, TTFields therapy essentially involves delivery of two incoherent electric fields to the tumor. A key question is how to meaningfully combine the two fields into a single metric defining dose. Ballo et al. established connections between local minimum field intensity (LMiFI) and local minimum power density (LMiPD) and survival. In this paper, dose was defined as LMiPD multiplied by usage.
} 


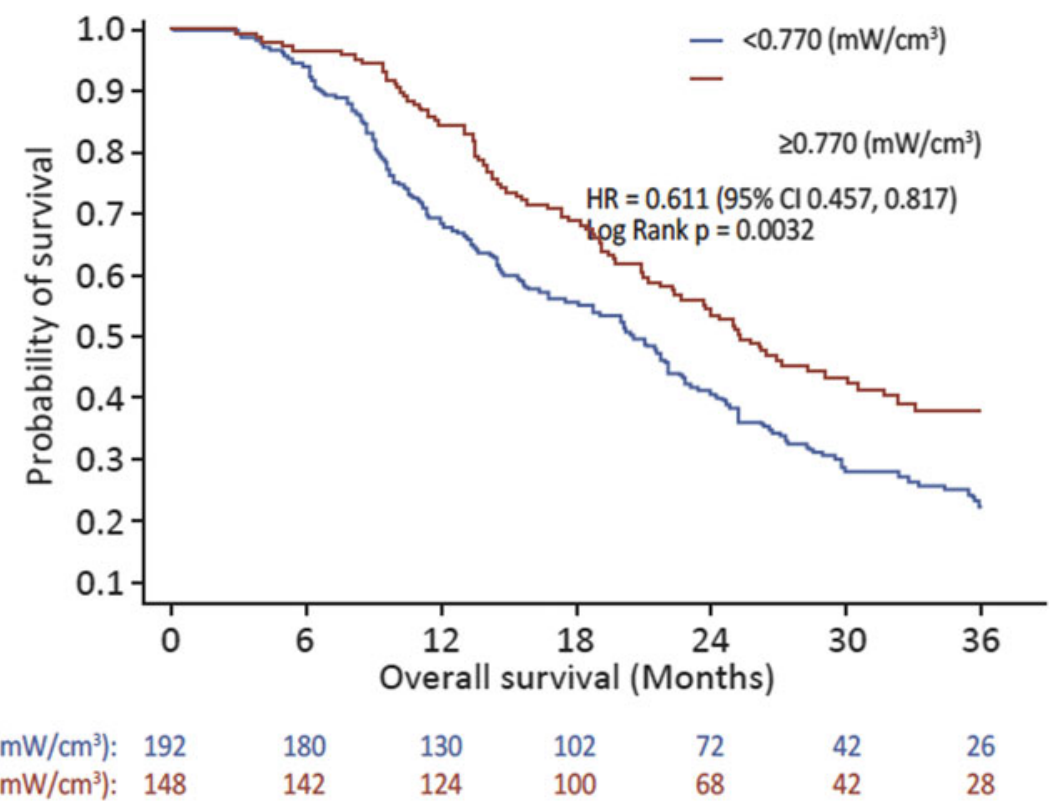

$\begin{array}{cccccccc}<0.770\left(\mathrm{~mW} / \mathrm{cm}^{3}\right): & 192 & 180 & 130 & 102 & 72 & 42 & 26 \\ \geq 0.770\left(\mathrm{~mW} / \mathrm{cm}^{3}\right): & 148 & 142 & 124 & 100 & 68 & 42 & 28 \\ & & & & & & & \\ & & \text { Patients } & \text { Event } & \text { Censored } & \text { Median OS } & 95 \% \mathrm{Cl} \\ <0.770\left(\mathrm{~mW} / \mathrm{cm}^{3}\right): & 192 & 150 & 42 & 20.43 & 16.63,22.73 \\ \geq 0.770\left(\mathrm{~mW} / \mathrm{cm}^{3}\right): & 148 & 96 & 52 & 25.23 & 21.70,30.47\end{array}$

Fig. 3 Kaplan-Meier curves showing overall survival for patients treated with TTFields during the EF-14 trial. Graph shows survival curves when patients are divided into two groups based on the average dose in the tumor bed. The graphs clearly show improved survival in the group of patients who received an average dose of above $0.77 \mathrm{~mW} / \mathrm{cm}^{3}$ at the tumor bed. (Graph adapted from Ballo et al. [2])

TTFields to the tumor bed does indeed improve OS. Thus, a first principle for TTFields treatment planning is that treatment planning should strive to maximize average dose delivered to a region comprising the tumor and the peritumoral region, analogous to radiation therapy planning. A major difference between radiation therapy planning and TTFields treatment planning is that due to the highly toxic nature of radiation therapy; radiation therapy plans also need to account for avoidance structures, in which the radiation dose should be limited. This adds a level of complexity to the planning process. TTFields has a very low toxicity profile, with the only adverse effect reported being skin toxicity $[19,22]$. Thus, there is no need to plan treatment to minimize dose in critical structures. It might be wise, however, to incorporate avoidance areas on the skin into the plan where arrays should not be placed.

Finally, it should be emphasized that TTFields treatment planning could benefit from understanding how the distribution of the field alters the progression of the tumor. This type of knowledge may help to devise more efficient strategies for 
planning the delivery of TTFields. These methods could be aimed at containing tumor growth by delivering higher doses to regions to which the tumor is more likely to grow or preventing tumor growth to critical structures by enhancing the field intensity in these regions.

\section{Patient-Specific Model Creation}

In order to properly perform numerical simulations of TTFields delivery, it is necessary to create accurate computational models that are patient-specific $[11,12$, 24-26]. This involves creating a 3D volume representing the patient, in which dielectric properties (primarily conductivity) are assigned to each voxel.

Two different approaches can be used for creating these models:

Segmenting medical images of the patients to identify the various tissue types in the model and assignment of typical conductivity values to each tissue type [26]

Mapping conductivity from imaging data to assign a conductivity value to each voxel in the patient model based on some signal in the imaging data that provides information about the dielectric properties at a point [26, 27]

To date, most modelling work associated with TTFields has relied on the segmentation of patient data and assignment of conductivity values to each tissue type. Conductivity values have been assigned to the tissues based on empirical measurements that appear in the literature. There is a high degree of certainty associated with the conductivity values reported for healthy tissues of the brain, as reported measurements are relatively consistent when comparing different reports. However, little to no information exists on the electric properties of brain tumors. As response to TTFields seems to depend on dose delivered to the tumor bed, and as dose to the tumor will be influenced by the electric properties of the tumor, it is important to gain reliable data on the electric properties of tumors. At EMBC 2019, Proescholdt et al. [17] presented data on this topic. The data relied on measurements performed on tissue probes acquired from 53 patients with tumors of different histology and malignancy grades: low-grade glioma $(n=5)$, glioblastoma (GBM; $n=16)$, meningioma $(n=19)$, brain metastases $(n=10)$, and other histology types (1 craniopharyngioma, 1 lymphoma, 1 neuroma). Tissue probes were acquired from the vital and perinecrotic compartments of the tumor if present. Several probes (up to five) were sampled from each region. Immediately after acquisition, the electric properties of tissue fragments taken from the probes were determined using a parallel plate setup. The impedance of the sample was recorded at frequencies $20 \mathrm{~Hz}-$ $1 \mathrm{MHz}$. These measurements revealed significant differences between the conductivity observed in different tumor types, with meningiomas showing the lowest conductivity (mean conductivity [S/m]: 0.189; range: $0.327-0.113$ ) and GBM tissue exhibiting the highest conductivity values (mean conductivity [S/m]: 0.382; range: 0.533-0.258). Consistently, the perinecrotic areas of tumors displayed lower conductivity values compared to the solid tumor compartments and also significant 
intratumoral heterogeneity in tumors of one specific histological diagnosis. The results of this study are summarized in Fig. 4.

This study sheds light on the dielectric properties of intracranial tumors, currently not accounted for in numerical models. An understanding of the cause of heterogeneity is needed in order to improve model quality and better predict field distributions around the tumor. In the interim, sensitivity analysis analyzing the effect of altering the electric properties of the tumor on field distributions is needed in order to complete our understanding on how best to plan TTFields therapy and the uncertainties associated with this planning.

EMBC 2019 also included a talk by Wenger et al. [26], discussing the use of water content-based electric property tomography (wEPT) in order to create patient models for TTFields-related numerical simulations. wEPT is an imaging tomography technique that models electrical conductivity, $\sigma$, and relative permittivity, $\varepsilon$, as monotonic functions of water content (WC) according to Maxwell's mixture theory [13]. WC maps are found via a transfer function mapping the image ratio (IR) of two T1w images with different repetition times (TR) into water content. Previously, wEPT was adapted to map WC, $\sigma$, and $\varepsilon$ at $200 \mathrm{kHz}$ in animal brain samples and tumor-bearing rats with mixed results [26]. When comparing wEPT-based predictions to empirical measurements of tissue samples using a parallel plate setup, we found a good match between wEPT-based estimations in the healthy tissue, while the quality of the match was poor within the tumors. At EMBC 2019, data were presented on the applicability of wEPT to mapping the electric properties of the human brain. The images used for wEPT mappings included, for this purpose, an image with a short TR resembling a conventional T1w MRI and a proton density (PD) image with the same parameters except for a long TR (Fig. 5).

EP maps for three patients who participated in the EF-14 trial were created using wEPT. The adapted wEPT model coefficients were found via curve fitting according to previous experiments and MRI scanner-specific parameters. Analysis of the results showed that wEPT estimates of WC, $\sigma$, and $\varepsilon$ in healthy brain tissues (white and gray matter) appear accurate and comparable with reports in literature. The properties were also relatively homogenous throughout the tissues and did not vary much between patients. Contrary, wEPT estimates of $\sigma$ and $\varepsilon$ in tumor tissues (necrosis, enhancing and non-enhancing tumor) were highly heterogeneous with high variability between patients.

These results, combined with results of our previous study, show the potential of wEPT-like methods for mapping the electric properties of the brain. However, the results suggest that wEPT alone is insufficient to map the electric properties of the tumor as well as the heterogeneous nature of the tumor. Future studies should focus on understanding the connection between tissue microstructure and the electric properties of the tissues at $200 \mathrm{kHz}$. When these processes are well understood, then methods for accurately mapping electric properties can be devised. 

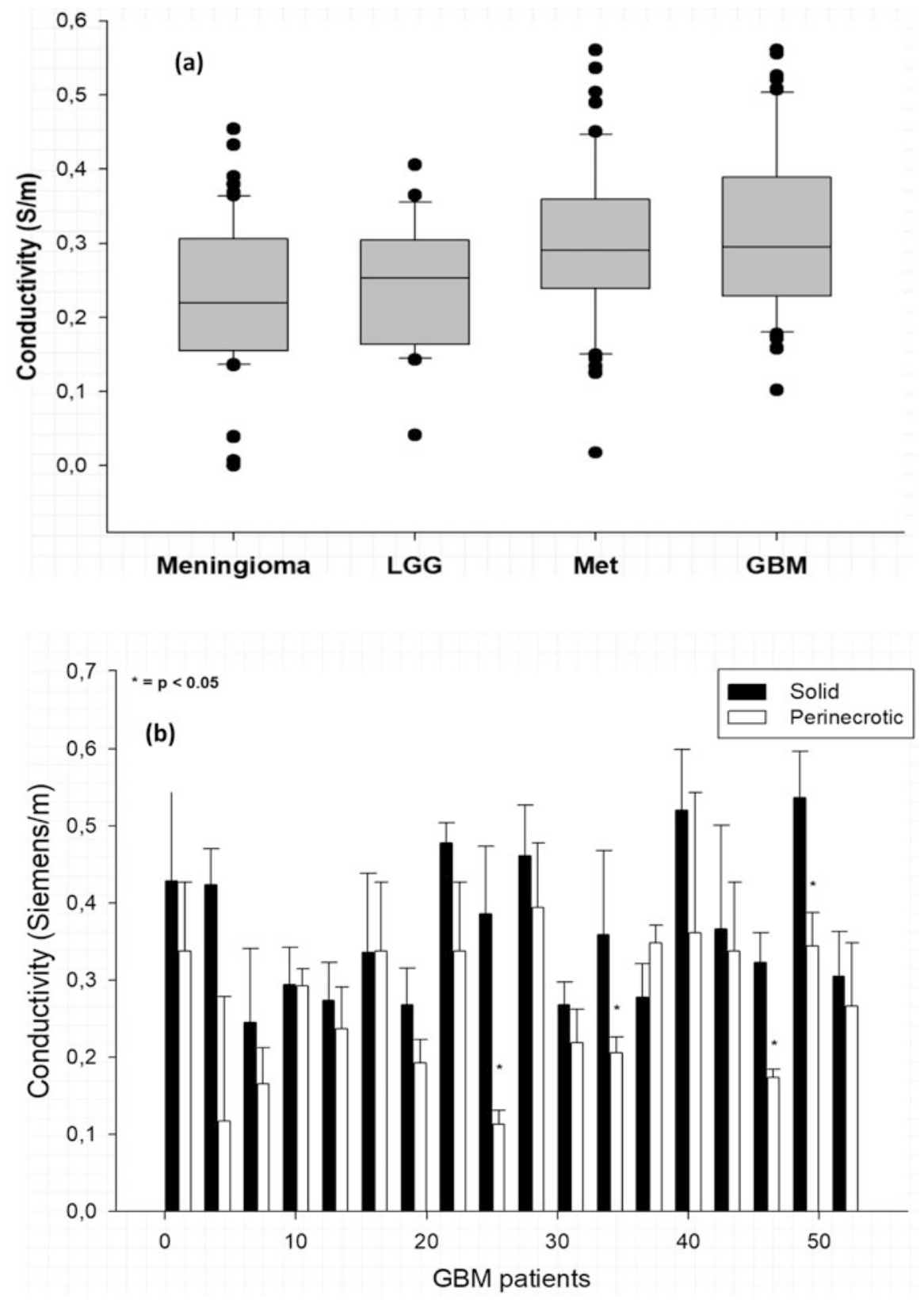

Fig. 4 (a) Boxplots showing the distribution of conductivities measured for four different brain tumor types: meningiomas, low-grade gliomas, brain metastases, and glioblastomas. Conductivity differs between tumor types, with the highest median conductivity measured in glioblastoma and the lowest median conductivity measured in meningioma. In all tumor types, a high heterogeneity in the electric conductivity is observed. (b) Bar plot showing the average conductivity measured in the solid and perinecrotic regions of the tumor for several GBM patients. Surprisingly, conductivity is consistently lower in the perinecrotic region of the tumor 

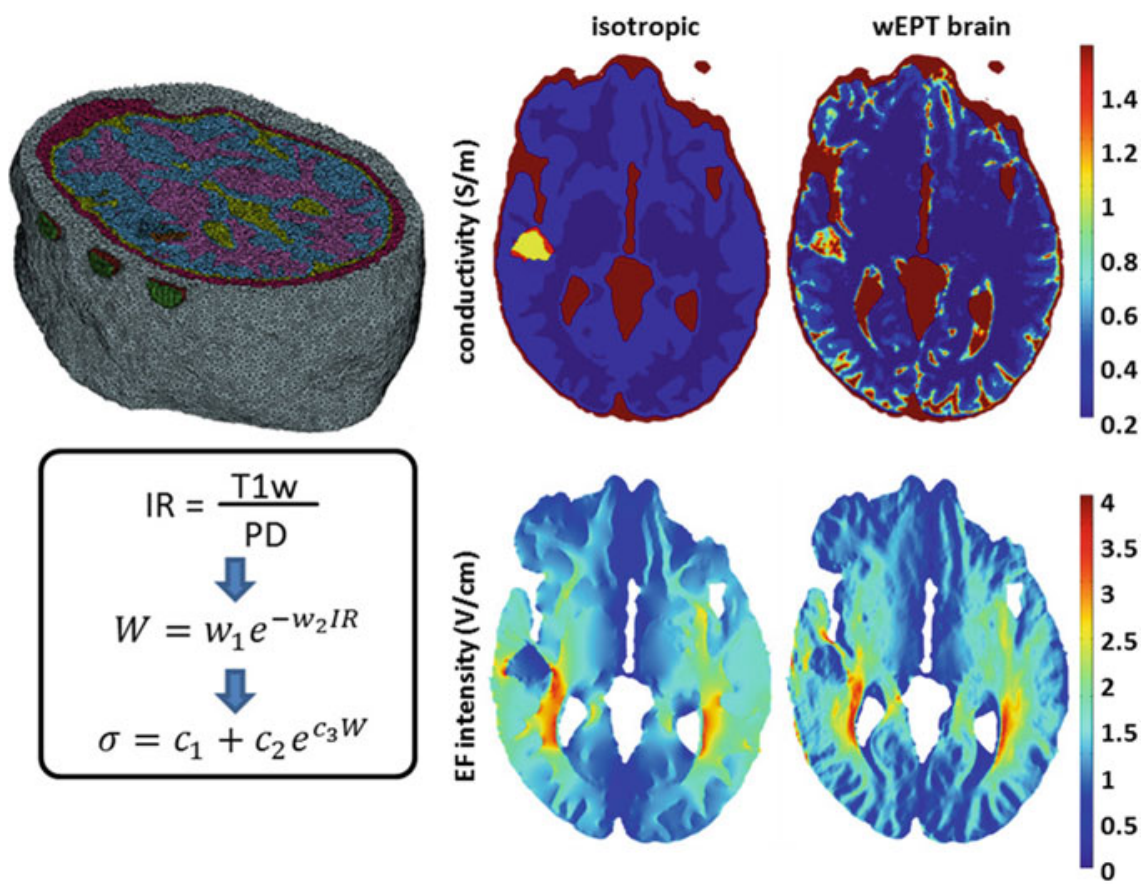

Fig. 5 (top-left) The tetrahedral mesh a of glioblastoma patient used to calculate TTFields induced electric field distributions, along with the (top row) distribution of conductivity for (top middle) a model created by segmenting a T1c image and assigning conductivity values to each tissue type and (top right) a model created using wEPT. Bottom row shows the field distribution in the (bottom middle) model created through segmentation and in the (bottom right) model created with wEPT. The flow chart in the bottom-left corner shows the wEPT scheme. First an image ration (IR) is calculated from the T1w and PD images. Next, WC is found from the IR, and conductivity derived from the WC. Note the parameters in the equations are found using curve-fitting to empirical data as detailed in Wenger et al. [26]

\section{Advanced Imaging for Monitoring Response to Therapy}

A key component in adaptive treatment planning is imaging. The ability to image the tumor and assess response effectively is key to mapping regions in which the tumor is responding to therapy and identifying the regions in which the disease is progressing. Treatment can then be adapted to target the regions of progression. Furthermore, advanced imaging holds the potential of early identification of molecular and biological responses occurring at the tissue and cellular level. These changes could indicate response or resistance to a specific treatment regimen, enabling the treating physician to adapt treatment early in order to improve the probability of positive outcomes. In the context of TTFields, this may mean adapting the transducer array position in order to increase the dose in regions of progression in order to suppress tumor growth in this region. 
At EMBC Mohan et al. presented a study investigating the use of advanced imaging to map response to TTFields [14]. Twelve patients (both newly diagnosed and recurrent GBM patients) previously treated with standard-of-care maximal safe resection and chemoradiation received TTFields. Patients underwent baseline (prior to initiation of TTFields therapy) and two follow-up ( 1 and 2 months post initiation of TTFields) image acquisitions on a 3 T MRI. DTI data were acquired using 30 directions with a single-shot spin-echo EPI sequence. Motion and eddy current corrections of raw DTI data were performed, and parametric maps of mean diffusivity (MD) and fractional anisotropy (FA) generated using in-house software. Perfusion-weighted imaging (PWI) was performed using T2*-weighted gradientecho EPI sequence which was acquired with a temporal resolution of $2.1 \mathrm{~s}$. Leakagecorrected cerebral blood volume (CBV) maps were constructed. 3D-EPSI was acquired using a spin-echo-based sequence. EPSI data were processed using the Metabolic Imaging and Data Analysis System (MIDAS) package. MD, FA, EPSI [choline (Cho)/creatine(Cr)], CBV maps, and FLAIR images were co-registered to post-contrast T1-weighted images, and contrast-enhancing neoplasms were segmented using a semiautomated algorithm. Median values of MD, FA, relative $\mathrm{CBV}$ (rCBV), and $\mathrm{Cho} / \mathrm{Cr}$ were computed at each time point, as were the 90th percentile rCBV (rCBVmax) values. Percent changes of each parameter between baseline and follow-up time points were evaluated.

Analysis of the images demonstrated an increasing trend in $\mathrm{MD}(\sim 3 \%)$ and declining trend in FA $(\sim 8 \%)$ at the 2-month follow-up relative to baseline. Additionally, reductions in $\mathrm{Cho} / \mathrm{Cr}$ and $\mathrm{rCBV}$ max from baseline to post-TTFields were also observed. All patients were clinically stable at 2-month follow-up. The changes in $\mathrm{MD}, \mathrm{FA}$, and $\mathrm{Cho} / \mathrm{Cr}$ may indicate inhibition of cellular growth. Reduction in rCBVmax may indicate anti-angiogenic effects associated with TTFields and decreased perfusion within the tumor bed after the therapy. These preliminary results suggest that advanced MR imaging may be useful in evaluating response to TTFields in GBM patients. Further work is required to validate the findings in a larger patient cohort in which these findings could be correlated with clinical endpoints of PFS and OS. Fully utilizing the power of these findings for TTFields treatment planning would also warrant studies looking to connect voxel-based changes observed in the images with field intensity distribution patterns derived from simulations. The completion of such studies would provide physicians with valuable information about how to plan and dynamically adapt TTFields dose distributions in order to maximize their ongoing effect on the tumor.

\section{Discussion and Conclusions}

In this chapter, we have provided an overview on some of the TTFields-related research presented at EMBC 2019. The research presented in this chapter relates to three key areas, which together lay the foundations for the field of TTFields dosimetry and treatment planning: 
- Definition of TTFields dose and the influence of dose on patient outcome

- The creation of patient-specific computational models for simulating delivery of TTFields

- Advanced imaging techniques for monitoring response to therapy

The area of TTFields dosimetry and treatment planning is very much in its infancy. The work presented at EMBC 2019 not only provides a basis for this field but also highlights the many open questions related to this field. The work presented by Bomzon et al. provides a robust and relatively intuitive definition for TTFields dose as the average power delivered by the fields. The authors clearly show a connection between TTFields dose at the tumor bed and patient survival. However, a crucial point required for effective treatment planning is to understand how dose distributions influence progression patterns. Do tumors really tend to progress to regions in which TTFields dose is lower? At a more fundamental level, given a TTFields dose distribution map, can we predict the probability that the tumor will progress in a certain region?

The work presented by Wenger et al. shows the potential of image-based electric property tomography to accurately map conductivity within patients, thereby providing a quick and accurate method for creating the patient-specific models required for TTFields treatment planning. This work also emphasizes the difficulty and knowledge gap that needs to be bridged in order to accurately map the electric properties of tissues in the vicinity of the tumor. The work by Proescholdt et al. shows that electric properties of tumors are indeed highly heterogeneous. Thus, accurate methods for modelling tumor tissue properties may be needed in order to accurately model electric field distributions in the vicinity of the tumor when performing treatment planning.

Finally, the work presented by Mohan shows the potential of advanced imaging techniques to identify metabolic and physiological changes within the tumor. These changes could be used as markers for response to therapy and could be adapted to plan therapy throughout the course of treatment.

Thus, the combination of work presented at EMBC poses key questions that need to be answered as the field of TTFields dosimetry and treatment planning evolves:

- How are progression patterns influenced by TTFields dose distributions? Do tumors progress in regions where dose is lower?

- How do we improve methods for mapping conductivity in a patient-specific method, specifically around the tumor?

- Can we utilize advanced imaging techniques to effectively monitor disease response/progression in order to better tailor therapy in an adaptive manner?

A final component required for the maturation of TTFields treatment planning is the development of a clinical quality assurance (QA) system analogous to that used for radiation therapy planning. This system should aim to establish the uncertainties associated with treatment plans and their effect on the dose distributions. This in turn could lead to the development of clinical guidelines related to the desired accuracy of the computational models used for TTFields treatment planning, the desired 
accuracy in the electric properties assigned to the various tissue types, and the desired accuracy in the placement of the transducer arrays on the skin when initiating treatment. Key to answering these questions are studies examining the sensitivity of numerical simulations of TTFields delivery to all of the above parameters, as well as studies aiming to experimentally validate the simulations using, for instance, suitable anthropomorphic phantoms. The development of this QA framework would enable the derivation of guidelines for best practices when performing TTFields treatment planning, thereby guiding the practicalities associated with the assimilation of sophisticated treatment planning procedures into the clinic.

As a concluding comment, we note that TTFields treatment planning could benefit enormously from emerging studies utilizing mathematical models to predict tumor progression $[1,7,8]$. These models attempt to incorporate information about factors such as tumor cell density, cell proliferation rates, and cell invasiveness into models that predict how tumors progress over time. Radiomic methods can be used to extract relevant information on the tumor, which can be fed into such models [5]. The effect of specific drugs or radiation therapy on tumor progression can then be modelled, and patients would gain potential benefit from a specific treatment quantified $[15,16]$. A natural expansion to these models is to incorporate TTFields dose distributions. In fact, an attempt to do this has previously been reported [18]. The benefit of different TTFields treatment plan can be evaluated, and the optimal plan selected. As the patient is monitored, and additional imaging data collected, the treatment plan, combination of therapies, and patient-specific model could be updated to continuously provide the patient with optimal care.

In summary, TTFields are emerging as a powerful addition to a growing arsenal of tools applied in the fight against cancer. The development of effective techniques for TTFields treatment planning will help to maximize the utility of this exciting treatment modality, ultimately leading to improved patient outcomes.

\section{References}

1. Alfonso, J., Talkenberger, K., Seifert, M., Klink, B., Hawkins-Daarud, A., Swanson, K., et al. (2017). The biology and mathematical modelling of glioma invasion: A review. Journal of the Royal Society, Interface, 14(136), 20170490.

2. Ballo, M., Urman, N., Lavy-Shahaf, G., Grewal, J., Bomzon, Z., \& Toms, S. (2019). Correlation of tumor treating fields dosimetry to survival outcomes in newly diagnosed glioblastoma: A large-scale numerical simulation-based analysis of data from the phase 3 EF-14 randomized trial. International Journal of Radiation Oncology Biology Physics, 104(5), 1106.

3. Bomzon, Z., Urman, N., Levi, S., Lavy-Shahaf, G., Toms, S., \& Matthew, B. (2019). Development of a framework for tumor treating fields dosimetry and treatment planning using computational phantoms (I). Berlin: EMBC.

4. Ceresoli, G., Aerts, J., Dziadziuszko, R., Ramlau, R., Cedres, S., van Meerbeeck, J., et al. (2019). Tumour treating fields in combination with pemetrexed and cisplatin or carboplatin as first-line treatment for unresectable malignant pleural mesothelioma (STELLAR): A multicentre, single-arm phase 2 trial. The Lancet Oncology, 20(12), 1702-1709. 
5. Gaw, N., Hawkins-Daarud, A., Hu, L., Yoon, H., Wang, L., Xu, Y., et al. (2019). Integration of machine learning and mechanistic models accurately predicts variation in cell density of glioblastoma using multiparametric MRI. Scientific Reports, 9(1), 10063.

6. Giladi, M., Schneiderman, R., Voloshin, T., Porat, Y., Munster, M., Blat, R., et al. (2015). Mitotic spindle disruption by alternating electric fields leads to improper chromosome segregation and mitotic catastrophe in cancer cells. Scientific Reports, 5, 18046.

7. Hawkins-Daarud, A., Johnston, S., \& Swanson, K. (2019). Quantifying uncertainty and robustness in a biomathematical model-based patient-specific response metric for glioblastoma. JCO Clinical Cancer Informatics, 3, 1-8.

8. Hu, L., Yoon, H., Eschbacher, J., Baxter, L., Dueck, A., Nespodzany, A., et al. (2019). Accurate patient-specific machine learning models of glioblastoma invasion using transfer learning. AJNR. American Journal of Neuroradiology, 40(3), 418-425.

9. Kirson, E., Dbalý, V., Tovaryš, F., Vymazal, J., Soustiel, J., Itzhaki, A., et al. (2007). Alternating electric fields arrest cell proliferation in animal tumor models and human brain tumors. Proceedings of the National Academy of Sciences of the United States of America, 104 (24), 10152-10157.

10. Kirson, E., Gurvich, Z., Schneiderman, R., Dekel, E., Itzhaki, A., Wasserman, Y., et al. (2004). Disruption of cancer cell replication by alternating electric fields. Cancer Research, 64(9), 3288-3295.

11. Korshoej, A., Hansen, F., Mikic, N., von Oettingen, G., Sørensen, J., \& Thielscher, A. (2018). Importance of electrode position for the distribution of tumor treating fields (TTFields) in a human brain. Identification of effective layouts through systematic analysis of array positions for multiple tumor locations. PLOS ONE, 13(8), e0201957.

12. Korshoej, A., Hansen, F., Thielscher, A., Von Oettingen, G., \& Sørensen, J. (2017). Impact of tumor position, conductivity distribution and tissue homogeneity on the distribution of tumor treating fields in a human brain: A computer modeling study. PLOS ONE, 12(6), e0179214.

13. Michel, E., Hernandez, D., \& Lee, S. (2017). Electrical conductivity and permittivity maps of brain tissues derived from water content based on T1-weighted acquisition. Magnetic Resonance in Medicine, 77(3), 1094-1103.

14. Mohan, S. (2019). Advanced imaging for monitoring response to TTFields in glioblastoma patients. Berlin: EMBC.

15. Neal, M., Trister, A., Ahn, S., Baldock, A., Bridge, C., Guyman, L., et al. (2013a). Response classification based on a minimal model of glioblastoma growth is prognostic for clinical outcomes and distinguishes progression from pseudoprogression. Cancer Research, 73(10), 2976-2986.

16. Neal, M., Trister, A., Cloke, T., Sodt, R., Ahn, S., Baldock, A., et al. (2013b). Discriminating survival outcomes in patients with glioblastoma using a simulation-based, patient-specific response metric. PLoS ONE, 8(1), e51951.

17. Proescholdt, M., Haj, A., Lohmeier, A., Stoerr Eva-Maria, E.-M., Eberl, P., Brawanski, A., et al. (2019). The dielectric properties of brain tumor tissue. Berlin: EMBC.

18. Raman, F., Scribner, E., Saut, O., Wenger, C., Colin, T., Fathallah-Shaykh, H.M. (2016). Computational Trials: Unraveling Motility Phenotypes, Progression Patterns, and Treatment Options for Glioblastoma Multiforme. PLoS One. 11(1), e0146617. https://doi.org/10.1371/ journal.pone.0146617PMCID: PMC4710507

19. Stupp, R., Taillibert, S., Kanner, A., Kesari, S., Steinberg, D., Toms, S., et al. (2015). Maintenance therapy with tumor-treating fields plus temozolomide vs temozolomide alone for glioblastoma a randomized clinical trial. JAMA: The Journal of the American Medical Association, 314(23), 2535-2543.

20. Stupp, R., Taillibert, S., Kanner, A., Read, W., Steinberg, D., Lhermitte, B., et al. (2017). Effect of tumor-treating fields plus maintenance temozolomide vs maintenance temozolomide alone on survival in patients with glioblastoma: A randomized clinical trial. JAMA, 318(23), $2306-2316$. 
21. Stupp, R., Wong, E., Kanner, A., Steinberg, D., Engelhard, H., Heidecke, V., et al. (2012). NovoTTF-100A versus physician's choice chemotherapy in recurrent glioblastoma: A randomised phase III trial of a novel treatment modality. European Journal of Cancer, 48 (14), 2192-2202.

22. Taphoorn, M., Dirven, L., Kanner, A., Lavy-Shahaf, G., Weinberg, U., Taillibert, S., et al. (2018). Influence of treatment with tumor-treating fields on health-related quality of life of patients with newly diagnosed glioblastoma a secondary analysis of a randomized clinical trial. JAMA Oncology, 4(4), 495-504.

23. Toms, S.A., Kim, C.Y., Nicholas, G., Ram, Z. (2019) Increased compliance with tumor treating fields therapy is prognostic for improved survival in the treatment of glioblastoma: a subgroup analysis of the EF-14 phase III trial. J Neurooncol. 141(2):467-473. Published online 2018 Dec 1. https://doi.org/10.1007/s11060-018-03057-zPMCID: PMC6342854.

24. Urman, N., Levy, S., Frenkel, A., Manzur, D., Hershkovich, H., Naveh, A., et al. (2019). Investigating the connection between tumor-treating fields distribution in the brain and glioblastoma patient outcomes. A simulation-based study utilizing a novel model creation technique. In N. Urman, S. Levy, A. Frenkel, D. Manzur, H. Hershkovich, A. Naveh, et al. (Eds.), Brain and human body modeling (pp. 139-154). Cham: Springer.

25. Wenger, C., Hershkovich, H., Tempel-Brami, C., Giladi, M., \& Bomzon, Z. (2019). Watercontent electrical property tomography (wEPT) for mapping brain tissue conductivity in the 200-1000 kHz range: Results of an animal study. In C. Wenger, H. Hershkovich, C. TempelBrami, M. Giladi, \& Z. Bomzon (Eds.), Brain and human body modeling (pp. 367-393). Cham: Springer.

26. Wenger, C., Miranda, P., Salvador, R., Thielscher, A., Bomzon, Z., Giladi, M., et al. (2018). A review on tumor-treating fields (TTFields): Clinical implications inferred from computational modeling. IEEE Reviews in Biomedical Engineering, 11, 195-207.

27. Wenger, C., Salvador, R., Basser, P., \& Miranda, P. (2016). Improving tumor treating fields treatment efficacy in patients with glioblastoma using personalized array layouts. International Journal of Radiation Oncology Biology Physics, 94, 1137.

Open Access This chapter is licensed under the terms of the Creative Commons Attribution 4.0 International License (http://creativecommons.org/licenses/by/4.0/), which permits use, sharing, adaptation, distribution and reproduction in any medium or format, as long as you give appropriate credit to the original author(s) and the source, provide a link to the Creative Commons license and indicate if changes were made.

The images or other third party material in this chapter are included in the chapter's Creative Commons license, unless indicated otherwise in a credit line to the material. If material is not included in the chapter's Creative Commons license and your intended use is not permitted by statutory regulation or exceeds the permitted use, you will need to obtain permission directly from the copyright holder. 